\title{
Evaluación del dolor en la punción de una fístula arteriovenosa para hemodiálisis usando pomada anestésica vs hielo
}

\author{
Patricia Ahis Tomás, Inma Peris Ambou, Carmen Ma Pérez Baylach, Joaquín Castelló Benavent
}

Centro de diálisis Nefrovall La Vall D' Uixò. Castellón

\section{Introducción:}

El paciente sometido a hemodiálisis lleva asociado a su enfermedad una gran comorbilidad que a menudo le causa dolor. Generalmente este dolor no es valorado en su totalidad y no se tiene en cuenta las limitaciones que provoca en la calidad de vida de estos pacientes. Diseñamos un estudio observacional analítico, que pretende evaluar el grado de dolor al que es sometido el paciente en hemodiálisis cuando se punciona su fístula arteriovenosa (FAVI). Para ello mediremos el dolor que produce dicha punción utilizando previamente uno de estos métodos: POMADA ANÉSTESICA (Emla®), PLACEBO (crema hidratante) o HIELO. Una vez tenemos medido el grado de dolor se compararán los resultados obtenidos con los tres métodos. En el caso de la pomada anestésica y la crema hidratante, el paciente no conoce cuál de las dos se ha utilizado a la hora de determinar el grado de dolor, para evitar sesgos en los resultados. También queremos evaluar si el método utilizado $(E m l a ®$, crema hidratante e hielo) interfiere en los parámetros de diálisis durante la sesión. Se eligió una muestra de 39 pacientes con enfermedad renal crónica, en tratamiento con hemodiálisis, pertenecientes al Centro de Diálisis Nefrovall (Castellón) y portadores de FAVI. Tras aplicar los criterios de exclusión, la muestra quedó reducida a 28 pacientes ( 23 hombres y 5 mujeres). Las variables medidas durante las tres semanas que duró el estudio fueron: dolor (escala EVA), flujo sanguíneo (FLUJO), presión arterial (PA), presión venosa (PV) y tiempo de coagulación (TCO). Se analizaron las 36 sesiones de hemodiálisis mediante el uso de una gráfica donde estaban registradas todas las variables y el uso del paquete estadístico SPSS. Se consideró estadísticamente significativo un $p$-valor $<0.05$. Por un lado concluimos que el método Pomada Anestésica ( $\mathrm{Emla}{ }^{\circledR}$ ) disminuye el dolor de la punción de forma estadísticamente significativa ( $p=0.004)$, siendo el método elegido por los pacientes en un $67.86 \%$. Por otro lado durante el uso de los tres métodos no se observan diferencias estadísticamente significativas en el resto de parámetros recogidos durante la sesión de hemodiálisis ( $P V p=0.191$, PA $p=0.278$, FLUJO $p=0.902$, TCO $p=0.445)$.

\section{$\underline{\text { Referencias Bibliográficas }}$}

1. Cañada R, Puig J, Ferrero S. Grado de dolor al pinchar la fístula arteriovenosa con agujas congeladas comparando con agujas a temperatura ambiente. Revista SEDEN, 2004; vol 7(2): 74-76.

2. Crespo R, Casas R, Muñoz J, Rivero F, Contreras $M^{a} D$, Muñoz L, López E. Influencia del calibre de la aguja sobre el grado de dolor originado en la punción de la fístula arteriovenosa.

3. García J, Sousa F, Rodríguez T, Andrés J, Alcalde $S$, Cardeñoso E, de la Cruz V, Hera R, Martín B, Ovejero C, Prieto V, Villacorta T, Carrera. Aplicación tópica de la lidocaína-prilocaína (emla) en pacientes en hemodiálisis ¿disminuye el dolor en la punción de la fístula?. Libro de comunicaciones del XXV Congreso de la SEDEN; Oviedo, 2000, 7-12.

4. Granados D, Abril F, Alcaraz N, Mañé J, Padilla J, Real M, Solano E. Una actuación de enfermería: intentar aliviar el dolor en las punciones de hemodiálisis. SEDEN. 2005; 8 (3): 231-236.

5. Maciá A, Pina JA. Recomendación de pomada anestésica emla en la punción a pacientes someti- 
dos a hemodiálisis periódica. Revista científica de Enfermería RECIEN, 2010,1989-6409.

6. Nilson A, Danielson K, Engberg G, Henneberg S. Ups J Med Sci 1990 (1): 87-94.

7. Watson A R, Szymkiw Pat, morgan A G. Topical Anaesthesia for fistula cannulation in haemodialy- sis patients. Nephrol Dial Transplant 1988, 3: 800802.

8. Wehle B, Björnström M, Cedgard M, Danielsson K, Ekernas A. Repeated application of EMLA cream $5 \%$ for the alleviation of cannulation pain in hemodialysis. Scand J Urul Nephology 1989. 23: 299302. 